\section{Spinal Epidural Hemangiomas: Various Types of MR Imaging Features With Histopathologic Correlation}

We read with interest the article by Lee et al ${ }^{1}$ in the August 2007 issue of the American Journal of Neuroradiology (AJNR), "Spinal Epidural Hemangiomas: Various Types of MR Imaging Features With Histopathologic Correlation." Over the past 25 years, there has been a quantum leap forward in our understanding of vascular anomalies, which has resulted in more accurate diagnosis, improved treatment, and focused research. This new-found clarity results from the fundamental distinction drawn between vascular tumors (the most common being infantile hemangioma) and vascular malformations, subclassified as capillary, venous, lymphatic, and arterial malformations (such as arteriovenous malformations and fistulas). ${ }^{2}$ This binary classification bears the imprimatur of the International Society for the Study of Vascular Anomalies. ${ }^{3}$ This nosology makes biologic and clinical sense, has been confirmed across a broad range of disciplines, and has led to the identification of several new clinical entities.

Inherited terminology, embedded deeply in our lexicon, is difficult to abandon. For example, what we commonly call a hepatic hemangioma in adults is, in fact, a venous malformation (rather than a vascular tumor of infancy that predictably regresses in early childhood). Nevertheless, the diagnostic impressions on abdominal MR and CT reports are unlikely to change anytime soon. Those of us in neuroradiology are equal offenders in the use of antiquated and confusing terminology, such as vertebral body "hemangiomas," noninfantile orbital "hemangiomas," and facial nerve "hemangiomas." These are all venous malformations, whereas "cystic hygromas" are lymphatic malformations. Nevertheless, terminological change is possible. For example, the old term cavernous hemangioma (implying tumor) has been replaced by the more accurate cerebral cavernous malformation (CCM). The CCM-related genes identified to date (CCM1, CCM2, and PDCD10) are involved in vascular and neuronal development, rather than tumor promotion or suppression.

Lee et al's article on intraspinal vascular anomalies specifically excludes intraosseous lesions, which are by common practice still referred to as intraosseous hemangiomas. ${ }^{4}$ Thus, the intraspinal soft tissue lesions described by the authors are not associated with an inherited tradition of inaccurate nomenclature. We were dismayed that the authors perpetuated the generic use of hemangioma and went on to distinguish subtypes, such as the "arteriovenous," the "cystlike" and the "solid hypervascular mass." This collection of lesions displays such a broad range of imaging characteristics because it is a lumping of disparate entities. To label both infantile hemangioma, which is fast-flow only in its proliferative stage, and the fast-flow of an arteriovenous malformation as "hemangioma of arteriovenous type" obscures the crucial fact that the infantile hemangioma very likely needs no treatment, whereas the arteriovenous malformation very likely does. Moreover, terminological inaccuracy has an impact on management: a problematic infantile hemangioma is likely to respond to corticosteroids, interferon, or vincristine, whereas these drugs have no role in treating arteriovenous malformations. Finally, the vascular biology underlying these lesions is vastly different. The endothelial marker glucose transporter 1 is positive in all stages of infantile hemangioma and negative in other vascular tumors and in vascular malformations. ${ }^{5}$ The histopathologic illustrations in this article predominantly exhibit findings characteristic of venous malformations.

We respectfully urge the editors of AJNR to guide authors on terminology and, in so doing, help move our specialty in the direction of more rational discourse with other disciplines involved in the care of vascular anomalies.

\section{References}

1. Lee JW, Cho EY, Hong SH, et al. Spinal epidural hemangiomas: various types of MR imaging features with histopathologic correlation. AJNR Am J Neuroradiol 2007;28:1242-48

2. Mulliken JB, Glowacki J. Hemangiomas and vascular malformations in infants and children: a classification based on endothelial characteristics. Plast Reconstr Surg 1982;69:412-22

3. Mulliken JB, Anupindi S, Ezekowitz RA, et al. Case records of the Massachusetts General Hospital. Case 13-2004: A newborn girl with a large cutaneous lesion, thrombocytopenia, and anemia. N Eng J Med 2004;350:1765-75

4. Greene AK, Rogers GF, and Mulliken JB. Intraosseous "hemangiomas" are malformations and not tumors. Plast Reconstr Surg 2007;119:1949-50.

5. North P, Waner M, Mizeracki A, et al. GLUT1: a newly discovered immunohistochemical marker for juvenile hemangiomas. Hum Pathol 2000;31:11-22.

D.B. Orbach

Neurointerventional Radiology Brigham and Women's Hospital

Boston, Mass

Vascular Anomalies Center Children's Hospital Boston

Boston, Mass

J.B. Mulliken

H.P.W. Kozakewich

Vascular Anomalies Center

Children's Hospital Boston

Boston, Mass

DOI 10.3174/ajnr.A0952 